\title{
Numerical Investigation of the Fluid Flow Characteristics in the Hub Plate Crown of a Centrifugal Pump
}

Wei Dong $^{1 *}$ and Wu-Li Chu ${ }^{1,2}$

\begin{abstract}
Due to the lack of understanding in the flow mechanism of the hub plate crown, the current calculation of the disc friction loss and the axial thrust in the centrifugal pump often uses empirical formulas. Research on the flow characteristics of the hub plate crown is of practical significance. The shroud and hub cavities are respectively studied with regard to tangential and radial velocities at the four different angular positions $\left(0^{\circ}, 90^{\circ}, 180^{\circ}\right.$, and $\left.270^{\circ}\right)$ at the four different operational points $\left(0.6 Q_{s p}, 0.8 Q_{s p}, 1.0 Q_{s p}\right.$, and $\left.1.2 Q_{s p}\right)$. Results indicate that at the same operational point, the smaller the volute chamber sectional area is, the higher the tangential velocity of the fluid core zone of the shroud cavity is. Radial leakage flow from the volute to the seal ring at the same operational point appears in $0^{\circ}$ and $90^{\circ}$ direction; when the flow is large, the tangential and radial velocities of the shroud and hub cavities with the same radius tend to be equal with axial symmetry. The axial leakage flow through the balance holes significantly affects the radial distribution of both tangential and radial velocities of fluid flow in the hub cavity. The numerical calculation results of fluid leakage through the clearance of back sealing ring are in good agreement with the test results. Accordingly, the magnitude of leakage is closely related to the fluid pressure and velocity distribution in the hub plate crown of the centrifugal pump. The analysis of the flow characteristics in the hub plate crown of the centrifugal pump could reveal the cause of the disc friction loss from the mechanism, providing a significant guidance for improving the accuracy of calculation and balancing the axial thrust in the centrifugal pump.
\end{abstract}

Keywords: Centrifugal pump, Shroud cavity, Hub cavity, Leakage of clearance, Numerical simulation

\section{Introduction}

In an impeller with balance holes of the double-sealing ring structure in a centrifugal pump, the hub plate crown of the impeller is divided into a shroud cavity and a hub cavity by the sealing ring. The calculation of round disk friction loss and axial thrust is closely related to the flow in the hub plate crown. Research on the shroud cavity, hub cavity, and sealing ring leakage has revealed the mechanism of the internal round disk friction loss and axial thrust of the centrifugal pump. Yang et al. [1] pointed out that disc friction loss calculated by the conventional method would produce more errors since it

\footnotetext{
*Correspondence: 735381559@qq.com

${ }^{1}$ School of Power and Energy, Northwestern Polytechnical University, Xi'an 710129, China

Full list of author information is available at the end of the article
}

only considered the friction torque of the impeller at its periphery but ignored the major part at the hub plate crown and sealing ring. In the derivation of the axial thrust calculation formula, under the assumption that no fluid leakage flow exists in the hub plate crown and the fluid rotational angular velocity within the shroud cavity is half of the impeller rotational angular velocity, no rotating motion of the hub cavity fluid exists within the sealing ring [2-4]. Salvadori et al. [5] pointed out that the cavity flow behavior is a key parameter for the pressure field evaluation and then for the thrust calculation. Over the pump operating range the main contribution to the axial thrust is due to the impellers flow fields, the leakage flows through sealing rings and the pressure distribution that occurs inside the cavities between impeller shrouds and pump stationary walls. Test results on disk friction loss or axial thrust calculated in terms of the theoretical 
formula in fluid flow velocity in the shroud cavity did not match the results of practical tests [6]. To calculate the axial thrust of centrifugal pump with higher accuracy, Liu et al. [7, 8] developed a mathematical model of the shroud cavity liquid pressure on the design condition by simplifying the Navier-Stokes equations for the movement of cavity fluid. The results of liquid pressure test verified the reliability of the model. The study of $\mathrm{Mu}$ et al. [9] showed that the pump clearance leakage flow has significant influence on the flow field in the hub plate crown. The angular velocity of the fluid in the back shroud cavity is less than 0.5 times the angular velocity of impeller. Besides, compared with the experiment result, the axial thrust was found underestimated by the conventional formula. Research has shown that both cavities velocity field in the shroud and hub and the axial changing laws in the centrifugal pump are similar to the velocity field in the enclosed cylinder body [10-12]. That is, in the fluid flow velocity of the shroud and hub cavities, a turbulent boundary layer exists near the outer wall of the rotating impeller back shroud. Near the internal wall of the fixed motionless pump shroud, a flow core zone exists along the axial direction without significant changes between two boundary layers [13-15]. The flow core zone is the main region of fluid within the shroud and hub cavities. It occupies over two-thirds of the axial distance, whereby the fluid flow conditions within the shroud and hub cavities can be reflected.

The fluid within the flow core zone of the hub plate crown of a centrifugal pump was regarded as the research object in this study. Based on a comparative analysis of the numerical results on pump performance and the test results, investigations were conducted on the flow of the hub plate crown at the $0.6 Q_{s p}, 0.8 Q_{s p}, 1.0 Q_{s p}$, and $1.2 Q_{s p}$ operational points to map the axial center fluid of the back shroud and hub cavities and the radial distribution curves of dimensionless tangential and radial velocities at the four different angular positions $\left(0^{\circ}, 90^{\circ}, 180^{\circ}\right.$, and $\left.270^{\circ}\right)$. The study was also aimed to analyze the radial distribution of the dimensionless tangential and radial velocities of the flow field in the back shroud and hub cavities. The test results of back sealing ring leakage of clearance are utilized to verify the accuracy and feasibility of the numerical research (Additional file 1).

\section{Research Models and Calculation Method}

\subsection{Research Models}

The main parameters of the centrifugal pump are as follows: flow rate $Q_{s p}=200 \mathrm{~m}^{3} / \mathrm{h}$, head $H=32 \mathrm{~m}$, efficiency $\eta=81 \%$, rotational speed $n=1450 \mathrm{r} / \mathrm{min}$, impeller outer diameter $D=325 \mathrm{~mm}$, sealing ring radial clearance $b=0.22 \mathrm{~mm}$, back sealing ring diameter $D_{m}=165 \mathrm{~mm}$, back sealing ring length $L=18 \mathrm{~mm}$, impeller hub diameter $D_{h}=60 \mathrm{~mm}$, balance hole diameter $d=8 \mathrm{~mm}$, and the number of balance holes and blades is six. A diagram of the back shroud cavity, hub cavity, and back sealing ring is shown in Figure 1.

Pro/E software was utilized to establish a complete geometric model of the flow channel. A section of the auxiliary computing domain was added to the impeller inlet and volute outlet to ensure that the outflow is fully developed and to erect it into a 3D model, as shown in Figure 2.

The back shroud cavity, the sealing ring clearance and the hub cavity are divided by structural grid in physical model of the centrifugal pump. The others are divided by nonstructural grid in the model. The structural grid classification pattern was adopted to improve the grid unit flow resolution. Grid independence test is presented, as shown in Figure 3. It can be noted that when the total number of elements are larger than $1.94 \times 10^{6}$, the efficiency do not significantly change. The numbers of elements are as follows: back shroud cavity is $8.81 \times 10^{5}$, hub cavity is $3.41 \times 10^{5}$, back sealing ring clearance is $2.29 \times 10^{4}$, and the total number of elements is $1.94 \times 10^{6}$. The distortion rate of all grid of the model is less than $89.36 \%$.

\subsection{Calculation Method}

Clean water was selected as the medium in the pump. The interfaces are created on impeller, volute, shroud cavity, sealing ring clearance, hub cavity, and balance holes

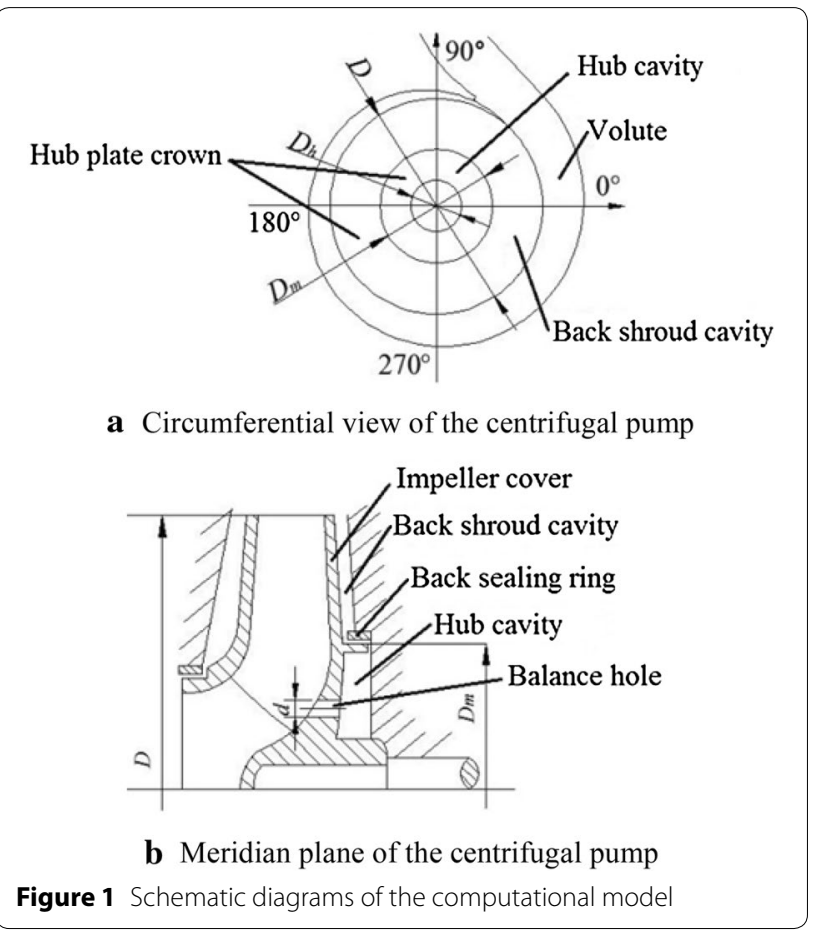




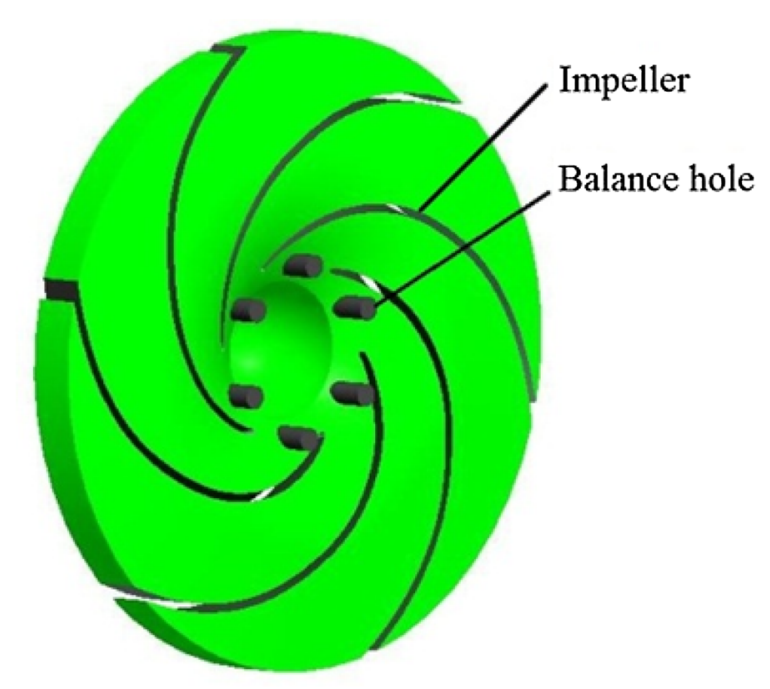

a Impeller and balance holes

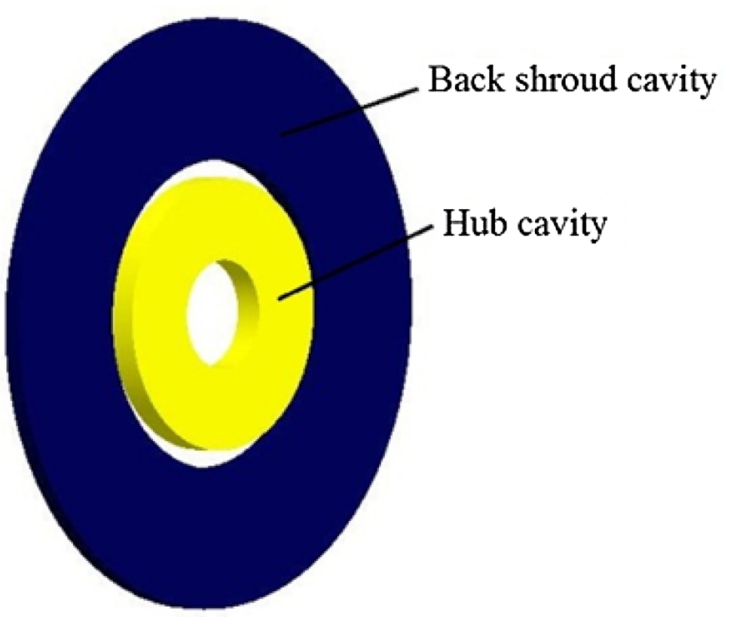

b Back shroud and hub cavities

Figure 2 Geometric model

with connection to the corresponding faces. Data can be effectively transmitted between different grid interfaces in the model. It forms circulating flow inside the pump. Boundary conditions were set as follows: the pump inlet was set as the velocity entrance without swirl flow, and the pump outlet was set as the free outflow pattern. Noslip condition was applied to the solid wall surfaces of the blade and volute, the wall function method was utilized to deal with the near-wall turbulence flow [16-18]. Given that the working medium in the centrifugal pump is clean water, the flow in the pump is incompressible turbulent flow with constant physical properties. Accordingly, the

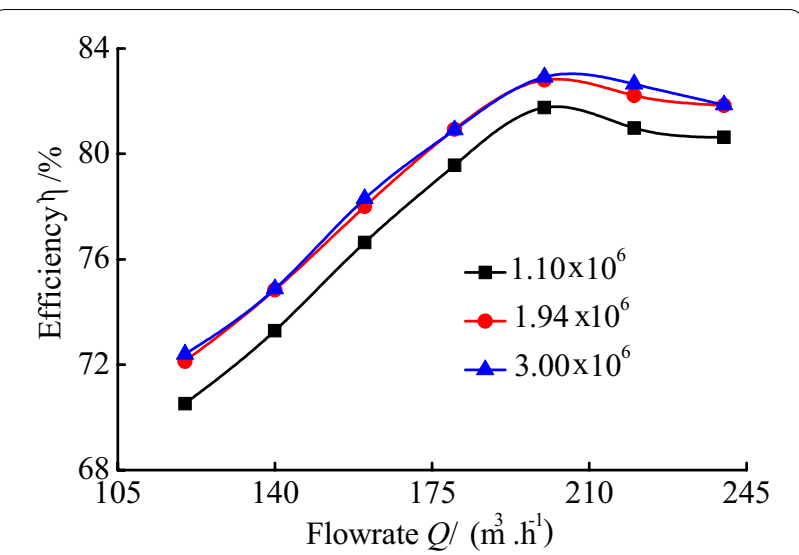

Figure 3 Grid independent verification

RNG $k-\varepsilon$ turbulence model was adopted, and a SIMPLEC algorithm was used to deal with the coupling between pressure and velocity [19-21]. Difference schemes were set as follows: the pressure sub-relaxation item is in the standard format, and the momentum sub-relaxation item, turbulent energy sub-relaxation item, turbulent energy dissipation sub-relaxation item, and turbulent energy dissipation rate are all Second-order Upwind Difference Scheme dispersion difference equations [22, 23]. Hence, numerical simulation can be conducted with FLUENT software. The impeller cover is set rotating wall in the hub plate crown, as shown in Figure 4.

\section{Numerical Method Validation}

\subsection{Performance Qualification}

The three-dimensional steady turbulent flow is simulated through the whole flow passage of the model. To ensure the reliability of the numerical calculation, the numerical simulation results were compared with the test results in

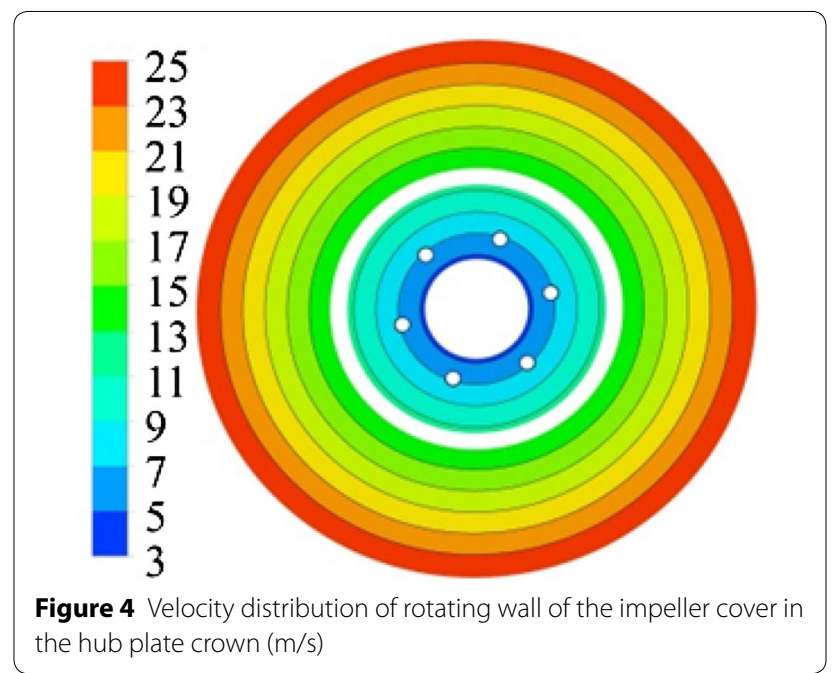




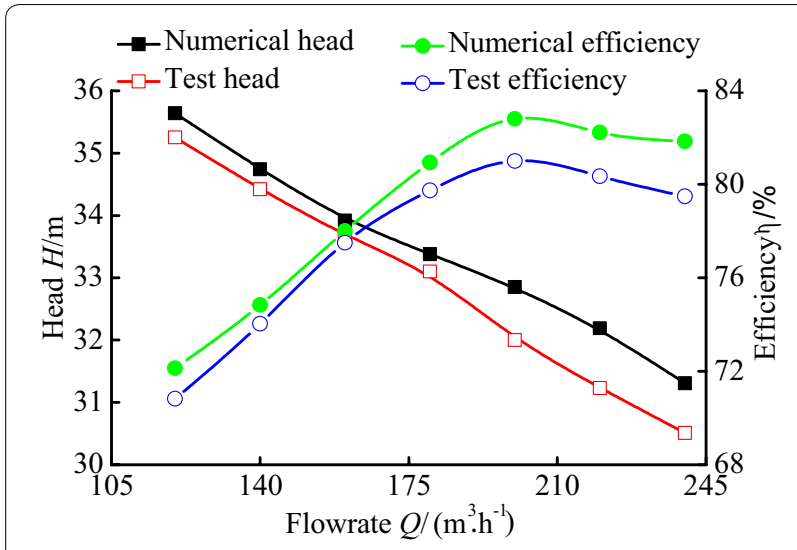

Figure 5 Performance curves of the centrifugal pump

terms of pump head and efficiency performance curves, as shown in Figure 5.

Figure 5 shows that under the operational conditions of volumetric flow rate ranging from $0.6 Q_{s p}$ to $1.2 Q_{s p}$, the relative errors of the numerical and test results on head and efficiency are within $2.98 \%$ and $2.88 \%$, respectively. The numerical simulation results on pump performance closely match the test results.

\subsection{Verification of Leakage in the Back Sealing Ring}

Given that the back sealing ring clearance can provide a break between the back shroud cavity and the hub cavity, a pressure difference exists between the back shroud cavity and the hub cavity. This difference could cause the fluid to leak from the high-pressure side to the low-pressure side through the back sealing ring clearance. Hence, the leakage of the back sealing ring clearance can reflect the side fluid pressure and its velocity distribution conditions in the back shroud to some extent. The fluid pressure difference before and after the sealing ring as well as continuity equation for the leakage flow are used in the traditional calculation method of leakage through the clearance of sealing ring to calculate the leakage [4]. In Ref. [24], the fluid leakage behavior test through the back sealing ring clearance was conducted on an enclosed test bed of pump. The procedure of combining pressure in the internal testing measure shroud cavity $\left(r_{1}=110 \mathrm{~mm}\right)$ and in the internal hub cavity $\left(r_{2}=62 \mathrm{~mm}\right)$ with the theoretical formula was adopted to calculate the leakage of the back sealing ring clearance in the centrifugal pump. A 1151-type Smart Pressure Transmitter was used to measure the pressure in the shroud and hub cavities. An LWGY-DN150-type Intelligent Turbine Flowmeter was utilized to determine the pump flow and display the realtime flow. The measurement error was less than $0.5 \%$. The measuring pattern of pressure designed is shown in Figure 6.

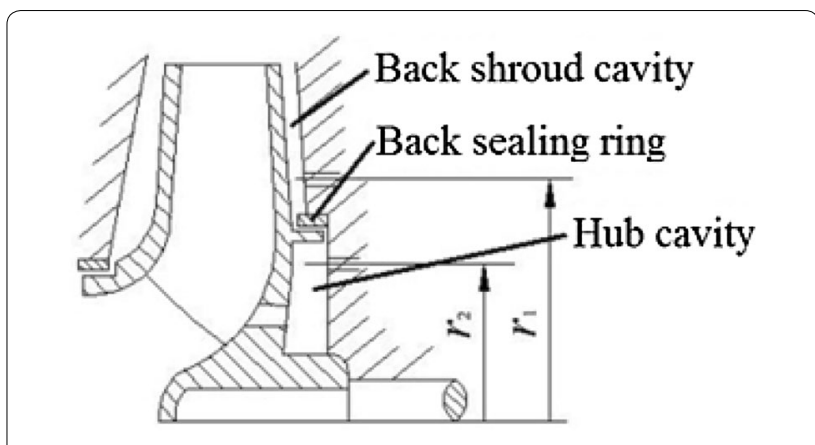

Figure 6 Sketch of fore-and-aft pressure test in the back sealing ring

Fluid leakage $q_{v}$ in the back sealing ring is as follows [24]:

$$
q_{v}=\psi D_{m} \pi b \sqrt{2 g \Delta H} .
$$

Flow coefficient $\psi$ in the sealing ring is as follows:

$$
\psi=\frac{1}{\sqrt{1+0.5 \xi+\frac{\lambda L}{2 b}}} .
$$

The fore-and-aft pressure difference $(\Delta H)$ in the back sealing ring is as follows:

$$
\Delta H=\frac{p_{1}}{\rho g}-\frac{p_{2}}{\rho g},
$$

where $p_{1} / \rho g$ is measured value at radius $r_{1}$ of the shroud cavity, $\mathrm{m} ; p_{2} / \rho g$ is measured value at radius $r_{2}$ of the hub cavity, $\mathrm{m} ; \xi$ is the shape coefficient, $\xi=0.28 ; \lambda$ is the resistance coefficient, $\lambda=0.05$.

In the working zone of the flow rate ranging from $0.6 Q_{s p}$ to $1.2 Q_{s p}$, numerical calculation can provide the fluid leakage through the clearance of the back sealing ring. The clearance fluid leakage in the back sealing ring can be converted into dimensionless coefficient $\bar{q}_{v}$, indicating that it occupies a percentage in the flow rate, $Q$, at an operational point. At the same time, the flow rate $Q$ at an operational point can be converted into a dimensionless coefficient $\bar{Q}$, which is the ratio of $Q$ to flow rate $Q_{s p}$ at the design operational point.

$$
\begin{aligned}
& \bar{q}_{v}=\frac{q_{v}}{Q} \times 100 \%, \\
& \bar{Q}=\frac{Q}{Q_{s p}} .
\end{aligned}
$$




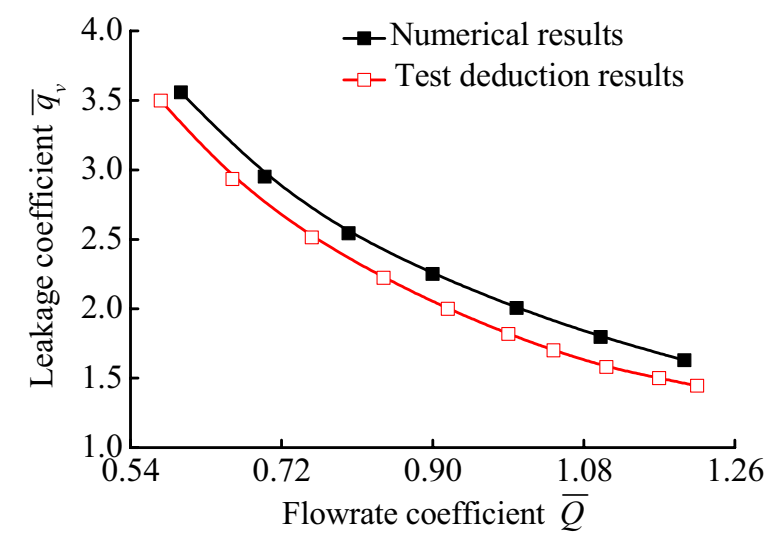

Figure 7 Comparison of the clearance fluid leakage rate in the back sealing ring

Thus, $\bar{q}_{v}=f(\bar{Q})$ relation curves can be obtained, as shown in Figure 7.

Figure 7 shows that the leakage rate obtained by numerical calculation is in agreement with that obtained by test deduction in terms of the changing tendency. When the flow rate increases, the pump head decreases, leading to a reduction of both the pressure difference at the inlet and outlet of the back sealing ring and the fluid leakage rate through the clearance. Given that the effect on vortex flow and fluid viscosity is neglected in the theoretical calculation formula, the results obtained by numerical calculation in the same flow operational point are always larger than those obtained by test deduction. However, the relative difference value remains basically unchanged. The relative difference between dimensionless simulation leakage rate and dimensionless test leakage rate is $0.19 \%$ in the flow rate, $1.0 Q_{s p}$, at the design operational point. For this reason, the numerical calculation results are in accordance with the test deduction results, thus proving that the research results of this study are accurate, and the numerical calculation method is feasible.

\subsection{Analysis of Leakage Flow in the Hub Plate Crown}

The fluid flow in the back shroud and hub cavities is similar to the fluid in the closed cylinder body, mainly consisting of radial pressure difference flow and tangential shearing flow, which can be considered as 2D viscous laminar flow [11-13]. At the same radial position or location, the fluid in the flow core zone does not undergo much change along the axial direction. The fluid radial flow behavior in the hub plate crown is formed because of the large kinetic energy at outlet of the impeller; the fluid flows into the back shroud cavity through the volute. In Ref. [25], the flow rate ranging from $0.6 Q_{s p}$ to $1.2 Q_{s p}$, numerical and experimental results of static pressure

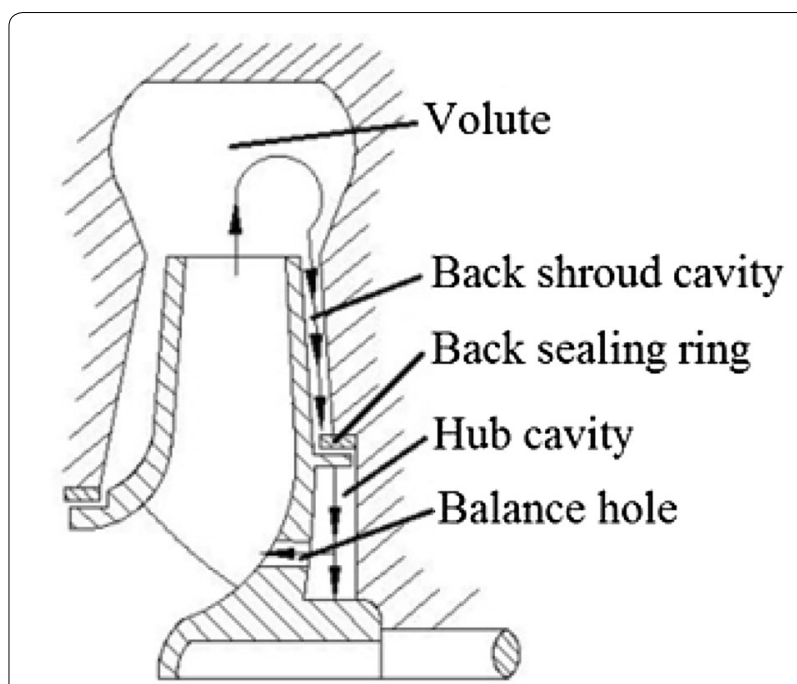

Figure 8 Sketch of the leakage flow of the hub plate crown in the centrifugal pump

are compared to verify the reliability of the numerical method in the back shroud cavity of the centrifugal pump. Under the action of radial pressure difference, the fluid flows from the back shroud cavity through the back sealing ring clearance into the hub cavity, leading to an increase in pressure potential energy of the fluid in the hub cavity. The fluid flows again through balance holes into the impeller. The leakage flow conditions in the hub plate crown are shown in Figure 8.

To verify the above argument, the fluid in the axial center position of the back shroud and hub cavities was regarded as the research object. Under the four operational conditions of $0.6 Q_{s p}, 0.8 Q_{s p}, 1.0 Q_{s p}$, and $1.2 Q_{s p}$, a detailed analysis was conducted on the radial distribution of the tangential and radial velocities in the back shroud cavity and the hub cavity at four different angular positions $\left(0^{\circ}, 90^{\circ}, 180^{\circ}\right.$, and $\left.270^{\circ}\right)$, thereby revealing the fluid flow behaviors in the back shroud and hub cavities.

For a convenient analysis, dimensionless tangential velocity $\bar{v}_{u}\left(\bar{v}_{u}=v_{u} / \omega r\right)$, dimensionless radial velocity $\bar{v}_{r}\left(\bar{v}_{r}=v_{r} / \omega r\right)$, and dimensionless radial length coefficient $\bar{r}(\bar{r}=2 r / D)$ were introduced, where $v_{u}$ is the fluid tangential velocity at the measuring point, $v_{r}$ is the fluid radial velocity at the measuring point, $\omega$ is the impeller rotational angular velocity, and $r$ is the radius at the measuring point.

\section{Calculation Results and Analysis}

\subsection{Velocity Distribution in the Back Shroud Cavity}

Given that little axial change occurred in the fluid of the flow core zone, the pump with $0.6 Q_{s p}, 0.8 Q_{s p}, 1.0 Q_{s p}$, and $1.2 Q_{s p}$ operational points was considered to analyze the distribution of fluid field. The cloud chart of velocity 


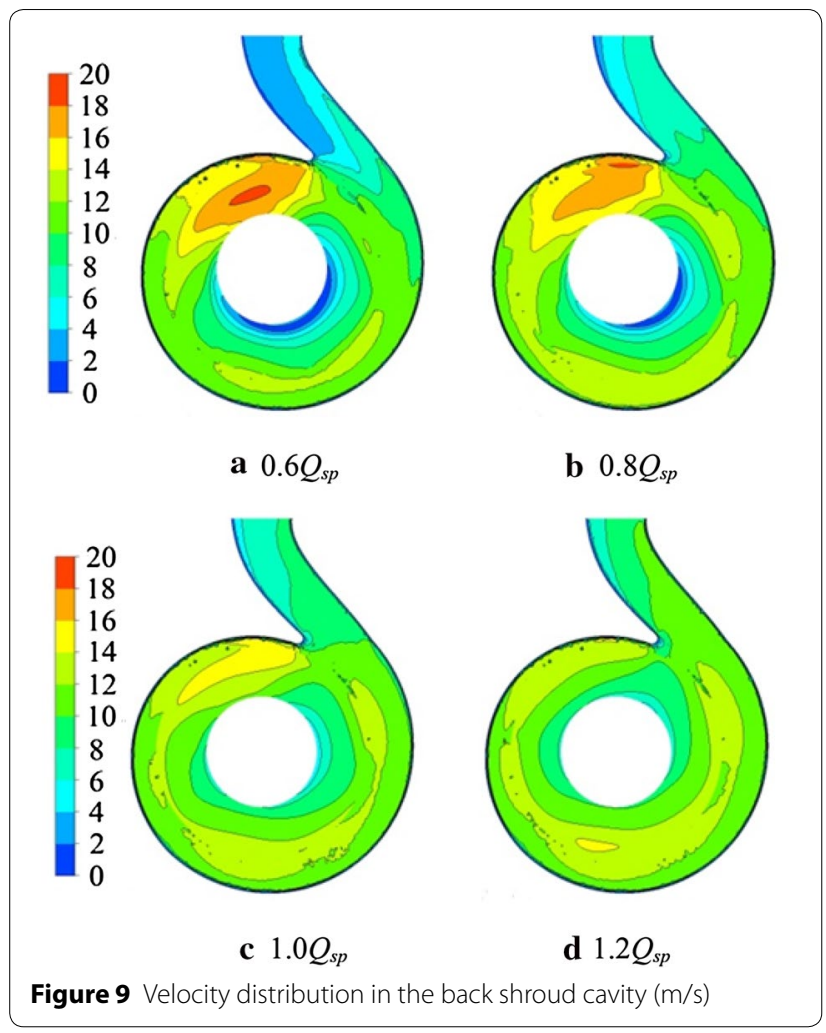

distribution in an axial center cross section of the back shroud cavity is shown in Figure 9.

Figure 9 shows that the fluid velocity in the shroud cavity is obviously subject to the effect of the main flow in the volute. Complicated tangential and radial changes in velocity occur without axial symmetry. When the flow increases from $0.6 Q_{s p}$ to $1.2 Q_{s p}$, the flow velocity in the high-speed zone near the volute tongue in the shroud cavity decreases, but the flow velocity in the low-speed zone near the sealing ring increases. Under the tangential and radial velocity of the fluid in the back shroud cavity, the fluid in the back shroud cavity at the four different angular positions $\left(0^{\circ}, 90^{\circ}, 180^{\circ}\right.$, and $\left.270^{\circ}\right)$ dimensionless tangential and radial velocity distribution can be mapped into curves, as shown in Figures 10 and 11.

Figures 10 and 11 indicate that in the same condition, a large difference in the radial distribution exists in the angles of $0^{\circ}, 90^{\circ}, 180^{\circ}$, and $270^{\circ}$ of the dimensionless tangential and radial velocities. The fluid in the flow core zone is in the circumference with the same radius, such that a significant change in the tangential and radial velocities occurs; however, near the volute, tangential and radial velocities are obviously subject to the constraint function of the main flow in the volute. Accordingly, the obtained value tends to be in agreement with an increase in radius. In the flow rate ranging from $0.6 Q_{s p}$ to $1.2 Q_{s p}$,

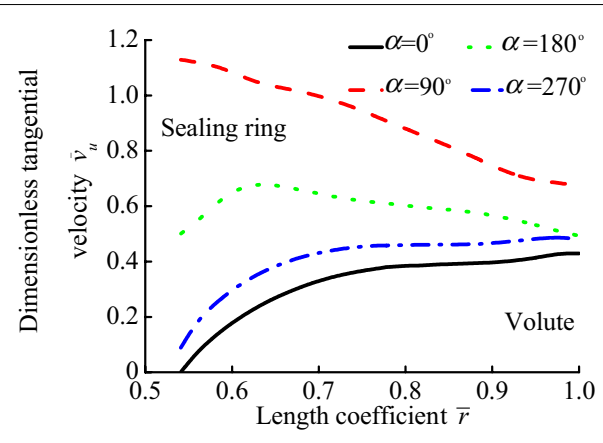

a $0.6 Q_{s p}$
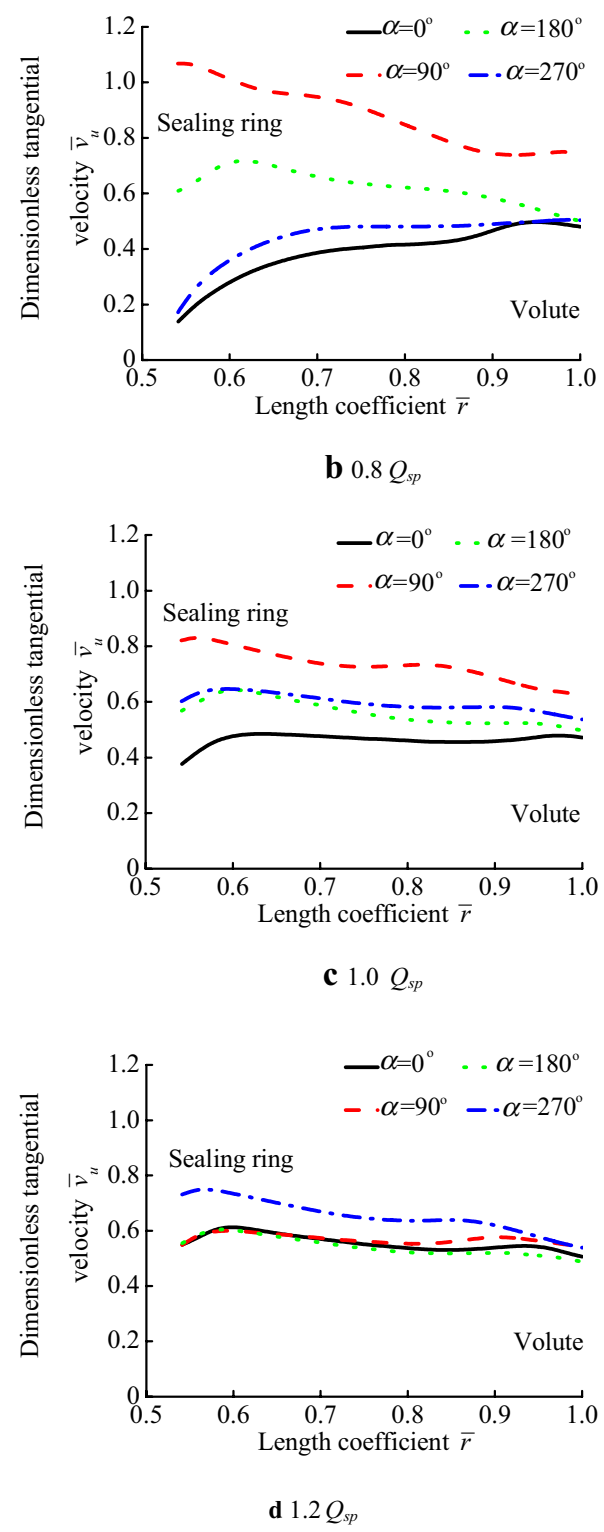

Figure 10 Radial distribution of dimensionless tangential velocity in the back shroud cavity 

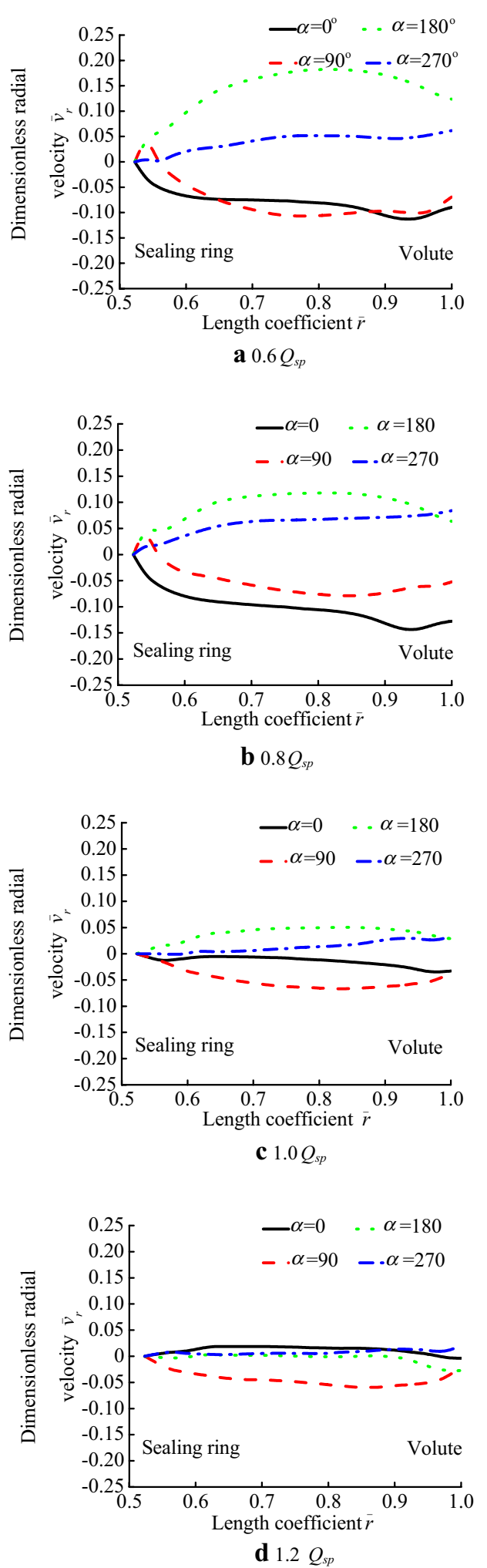

Figure 11 Radial distribution of dimensionless radial velocity in the back shroud cavity the larger the flow is, the more nearly equal the magnitude of dimensionless tangential and radial velocities with the four angles tends to become. Above the degree of $0^{\circ}$, $\bar{v}_{u}$ increases with an increase in radius. The larger the flow becomes, the larger $\bar{v}_{u}$ is; however, $\bar{v}_{r}$ is smaller than zero. That is, the fluid flows from the volute to the sealing ring. Above the degree of $90^{\circ}, \bar{v}_{u}$ decreases with an increase in radius, and the flow becomes large. $\bar{v}_{u}$ is small, but $\bar{v}_{r}$ is always smaller than zero, that is, the fluid flows from the volute to the sealing ring. Above the degree of $180^{\circ}, \bar{v}_{u}$ is small with the change in the radius and flow rate. Meanwhile, $\bar{v}_{u}$ is always larger than 0.5 , and $\bar{v}_{r}$ is always larger than zero. At the same radius, $\bar{v}_{r}$ becomes small with an increase in flow, that is, the fluid flows from the sealing ring to the volute. The larger the flow is, the smaller $\bar{v}_{r}$ is. Above the degree of $270^{\circ}, \bar{v}_{u}$ increases in radius. The larger the flow rate is, the larger $\bar{v}_{u}$ is. However, $\bar{v}_{r}$ is always close to zero, that is, the fluid is approximately still along the radial direction. The above phenomena are due to the fact that the high-energy fluid in the impeller outlet flows into the back shroud cavity through the volute. The high-energy fluid in the impeller outlet continues to transfer energy to the low-energy fluid in the back shroud cavity with an inner radius. The cross-sectional area of the volute cross section with $90^{\circ}$ angle direction is small, and the energy obtained in the inner radius increases rapidly. However, the radial pressure difference increases with increased dynamic energy. The cross-sectional area of the volute cross section with $0^{\circ}$ angle direction is large, but the radial pressure difference is small. The fluid in the inner radius has less dynamic energy. Hence, the larger the cross-sectional area of the volute cross section is, the smaller the $\bar{v}_{u}$ of the fluid in the back shroud cavity is. The $\bar{v}_{r}$ radial distribution condition illustrates that the existence of $0^{\circ}$ and $90^{\circ}$ fluid in the core zone of the back shroud cavity signifies a radial flow from the volute to the sealing ring, that is, leakage flow exists. An increase in flow operation and in the main fluid flowing in the volute may exert a significant impact on the fluid flowing in the shroud cavity; therefore, the value of tangential and radial velocities tends to be in agreement. The main flow direction is also agreement with Figure 8.

\subsection{Velocity Distribution in the Hub Cavity}

Equally, the cloud chart of fluid flow velocity distribution in an axial center cross section of the hub cavity is cut off, as shown in Figure 12.

Figure 12 shows that the fluid velocity in the hub cavity increases with an increase in flow rate ranging from $0.6 Q_{s p}$ to $1.2 Q_{s p}$. At the $0.6 Q_{s p}$ and $0.8 Q_{s p}$ small-flow operational points, a high-speed vortex flow zone exists near the inner radius in the hub cavity owing to the common influence of the hub rotational movement and 


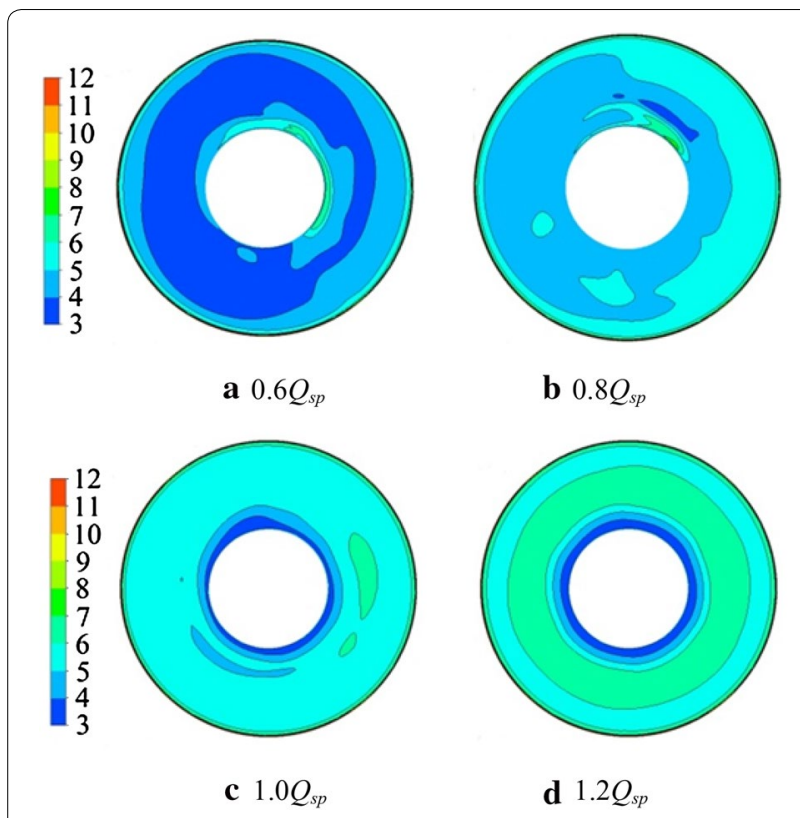

Figure 12 Velocity distribution in the hub cavity $(\mathrm{m} / \mathrm{s})$

the axial leakage flow in the balance holes. At the $1.2 Q_{s p}$ large-flow operational point, the fluid flow velocity in the hub cavity exhibits the changing trend of increasing first and then decreasing with an increase in radius. At the outer radius of the hub cavity, a large velocityincreasing zone with an extremely thin velocity gradient is formed because of the rotational movement of the interface of the sealing ring. The curves of the relationship between fluid tangential and radial velocities and radius in the hub cavity axial center are shown in Figures 13 and 14.

Figures 13 and 14 indicate that at the same flow operational points and owing to the rotational movement of the sealing ring interface and hub, a radial velocityincreasing boundary zone is formed at the two ends of dimensionless tangential velocity $\bar{v}_{u}$. Meanwhile, a lessthan-zero zone exists at dimensionless radial velocity $\bar{v}_{r}$, that is, flowing from the sealing ring to the hub. Apart from one boundary at the two ends, when $\bar{r}>0.3$, the $\bar{v}_{u}$ of the four angles decreases with an increase in radius, and $\bar{v}_{r}$ tends to become zero. When $\bar{r}<0.3$, a large difference exists in $0^{\circ}, 90^{\circ}, 180^{\circ}$, and $270^{\circ}$ angles. This is because the balance holes are located at the 0.24 to 0.3 radius of the impeller. The axial leakage flow through the balance hole exerts a certain effect on the tangential velocity distribution of the hub cavity, leading to an increase of the $\bar{v}_{u}$ at radial zone of the balance hole position. The larger the flow is, the more obvious the $\bar{v}_{u}$ increase is. The $\bar{v}_{r}$
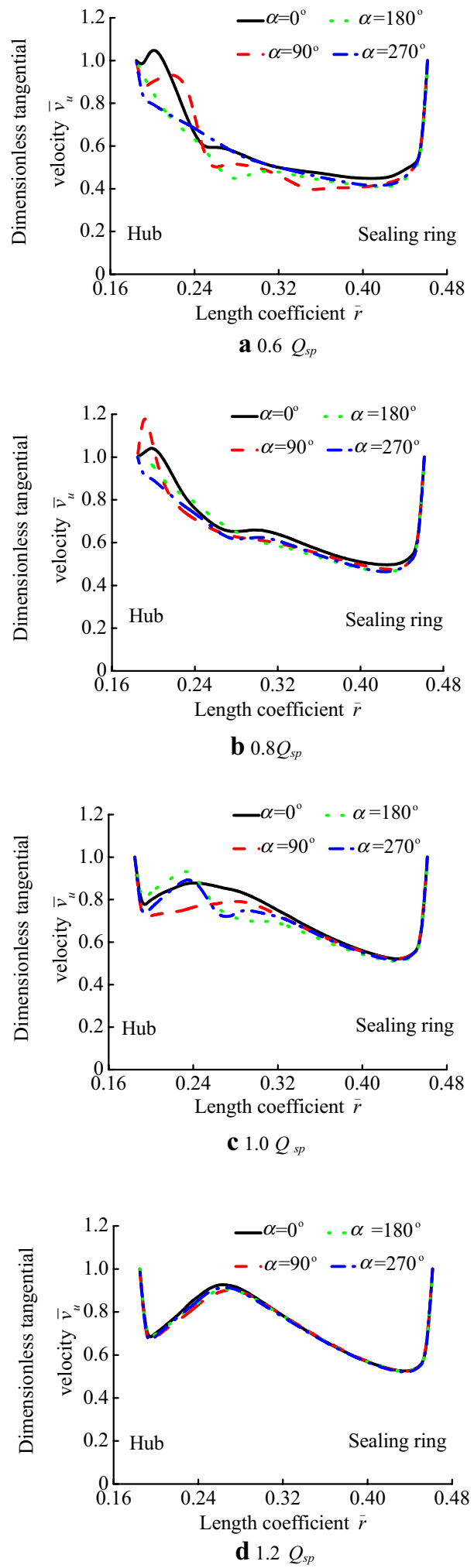

Figure 13 Radial distribution of dimensionless tangential velocity in the hub cavity 

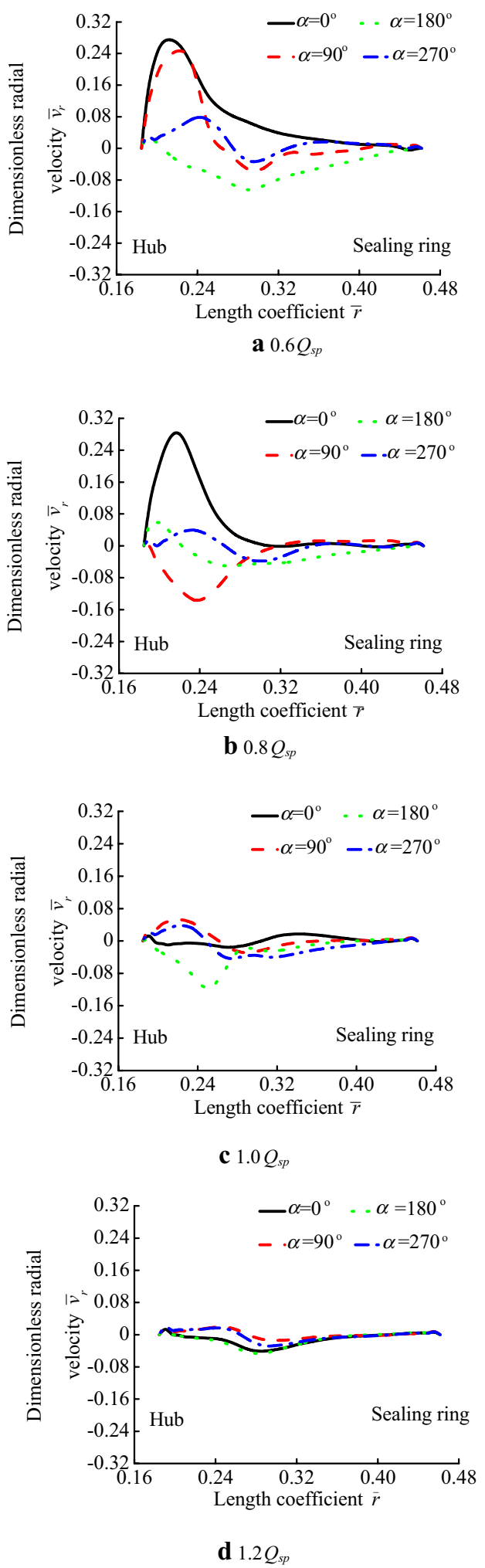

Figure 14 Radial distribution of dimensionless radial velocity in the hub cavity distribution in the four angles of $0^{\circ}, 90^{\circ}, 180^{\circ}$, and $270^{\circ}$ is in less agreement than that in the rest of the other zones.

Figures 13 and 14 also show that when the flow rate increases from $0.6 Q_{s p}$ to $1.2 Q_{s p}$, the value of dimensionless tangential velocity $\bar{v}_{u}$ and dimensionless radial velocity $\bar{v}_{r}$ at the same angles of $0^{\circ}, 90^{\circ}, 180^{\circ}$, and $270^{\circ}$ tends to be equal. At the $0.6 Q_{s p}$ and $0.8 Q_{s p}$ small-flow operational points, the hub nearby and balance hole zones $\bar{v}_{u}$ and $\bar{v}_{r}$ exhibit a large variation at the angles of $0^{\circ}, 90^{\circ}$, $180^{\circ}$, and $270^{\circ}$. When $\bar{r}>0.3, \bar{v}_{r}$ tends to be nearly zero, thereby illustrating that the hub rotational movement and axial leakage flow through the balance hole can cause a vortex movement, which in turn exerts a significant impact on small flow tangential and radial velocities distributed along the circumference. At the $1.0 Q_{s p}$ and $1.2 Q_{s p}$ operational points, $\bar{v}_{u}$ increases with an increase in radius, resulting in velocity reduction near the hub. In the balance hole zone, the velocity increases first and then decreases. In the process of velocity increase near the sealing ring, $\bar{v}_{r}$ always tends to be almost zero. Particularly at the $1.2 Q_{s p}$ large-flow operational point, the distribution of fluid velocity in the hub cavity is of axial symmetry.

\section{Conclusions}

(1) The magnitude of clearance leakage rate in the impeller sealing ring can reflect the flow conditions in the hub plate crown and the radial distribution of pressure and velocity in the back shroud and hub cavities in the centrifugal pump.

(2) At the same flow operational point, the smaller the cross-sectional area of the volute is, the larger the fluid tangential velocity of the flow core zone in the back shroud cavity is. At angles of $0^{\circ}$ and $90^{\circ}$, radial leakage flow occurs from the volute to the sealing ring.

(3) The main flow in the volute exerts a limited effect on the fluid flowing in the shroud cavity. When the flow increases from $0.6 Q_{s p}$ to $1.2 Q_{s p}$, the tangential and radial velocities at the position with the same radius gradually tend to be equal.

(4) The joint action of hub rotational movement and axial leakage flow through the balance hole exerts significant effects on the radial distribution of the tangential and radial velocities of the fluid flowing in the hub cavity. The larger the flow is, the more axial symmetry exists in the fluid flow velocity distribution in the hub cavity. 


\section{Additional file}

Additional file 1. Brief introduction of the article.

\section{Authors' contributions}

WLC was in charge of the whole trial; WD wrote the manuscript; WD assisted with sampling and laboratory analyses. Both authors read and approved the final manuscript.

\section{Author details}

${ }^{1}$ School of Power and Energy, Northwestern Polytechnical University, Xi'an 710129, China. ${ }^{2}$ Collaborative Innovation Center of Advanced AeroEngine, Beijing 100191, China.

\section{Authors' Information}

Wei Dong, born in 1987, is currently a PhD candidate at School of Power and Energy, Northwestern Polytechnical University, China. He received his master degree from Lanzhou University of Technology, China, in 2014. His research interests include flow theory, test and control of fluid machinery.

Wu-Li Chu, born in 1962, is currently a professor and a PhD candidate supervisor at School of Power and Energy, Northwestern Polytechnical University, China. His main research interests include aerothermodynamic of turbomachinery.

\section{Competing Interests}

The authors declare that they have no competing interests.

\section{Funding}

Supported by National Natural Science Foundation of China (Grant Nos. 51576162, 51236006).

\section{Publisher's Note}

Springer Nature remains neutral with regard to jurisdictional claims in published maps and institutional affiliations.

Received: 8 August 2016 Accepted: 6 August 2018

Published online: 16 August 2018

\section{References}

[1] CXYang, C Qian. Calculation of disc loss in centrifugal pump with low specific speed. Journal of Lanzhou University of Technology, 2012, 38(3): 56-60. (in Chinese)

[2] T Shimura, S Kawasaki, M Uchiumi, et al. Internal flow and axial thrust balancing of a rocket pump. Journal of Fluid Engineering, Transactions of the ASME, 2012, 134(4): 041103-1-041103-8.

[3] W D Shi, Q F Li, W G Lu, et al. Estimation and experiment of axial thrust in centrifugal pump based on CFD. Transactions of the Chinese Society for Agricultural Machinery, 2009, 40(1): 60-63. (in Chinese)

[4] X F Guan. Theory and design of modern pump. Beijing: China Astronautic Publishing House, 2011. (in Chinese)

[5] S Salvadori, A Marini, F Martelli. Methodology for the residual axial thrust evaluation in multistage centrifugal pumps. Engineering Applications of Computational Fluid Mechanics, 2012, 6(2): 271-284.

[6] Y J He, G F Zhou, J Q Pan, et al. Experiment for axial thrust of multi-stage pump for seawater desalination. Drainage and Irrigation Machinery, 2009, 27(2): 105-110. (in Chinese)

[7] Z L Liu, S Zhang, A C Shao, et al. Theoretical computation and verification for fluid static pressure in centrifugal pump side chamber. Journal of Mechanical Engineering, 2016, 52(4): 178-184. (in Chinese)
[8] Z L Liu, W Dong, N Zhang, et al. Calculation and validation of fluid pressure of balance cavity in centrifugal pump. Transactions of the Chinese Society for Agricultural Engineering, 2013, 29(20): 54-59. (in Chinese)

[9] J G Mu, S Li, S H Zheng, et al. Influence of interstage leakage on the axial force of the multistage centrifugal pump. Transactions of the Chinese Society for Agricultural Machinery, 2010, 41(7): 40-44. (in Chinese)

[10] J H Yang, C L Wang, J P Li. Mathematical model of flow inside a centrifugal pump casing. Transactions of the Chinese Society for Agricultural Machinery, 2003, 34(6): 68-72. (in Chinese)

[11] XY Wang, C X Wang, Y B Li. Numerical study of slow characteristics in the impeller side chamber of centrifugal pump. Transactions of the Chinese Society for Agricultural Machinery, 2009, 40(4): 86-90. (in Chinese)

[12] SH Park, G L Morrison. Analysis of the flow between the impeller and pump casing back face for a centrifugal pump. Proceedings of the ASME Fluids Engineering Division Summer Meeting, Vail, Colorado, USA, August 2-6, 2009: 221-235.

[13] D Lefor, J Kowalski, B Kutschelis, et al. Optimization of axial thrust balancing swirl breakers in a centrifugal pump using stochastic methods. Proceedings of the ASME 2014 4th Joint US-European Fluids Engineering Division Summer Meeting (FEDSM2014-21262), Chicago, Illinois, USA, August 3-7, 2014: 1-11.

[14] J F Gülich. Disk friction losses of closed turbomachine impellers. Forschung im Ingenieurwesen/Engineering Research 2003, 68(2): 87-95.

[15] D Y Pan, T Wang, B Zhang, et al. PIV measurement on rotating disks flow in cylinder. Chinese Journal of Hydrodynamics, 2009, 24(2): 200-206. (in Chinese)

[16] W Dong, W L Chu. Influence of balance hole diameter on performance and balance chamber pressure of centrifugal pump. Transactions of the Chinese Society for Agricultural Machinery, 2015, 46(6): 73-77. (in Chinese)

[17] M G Tan, S Q Yuan, H L Liu, et al. Numerical research on performance prediction for centrifugal pumps. Chinese Journal of Mechanical Engineering, 2010, 23(1): 21-26.

[18] W D Cao, X Dai, Q X Hu. Research on the effect of impeller reflux balance holes on pressure and axial force of centrifugal pump. Proceedings of the ASME 2014 4th Joint US-European Fluids Engineering Division Summer Meeting (FEDSM2014-21992), Chicago, Illinois, USA, August 3-7, 2014: 1-12.

[19] Q S Wei, Z L Liu. Numerical analysis and validation of floating impeller balance chamber pressure in centrifugal pump based on CFD. Proceedings of the CSEE, 2011, 31(14): 103-108. (in Chinese)

[20] L S Zhang, J Jiang, Z H Xiao, et al. Numerical investigation of the effect of balancing-hole on the axial force of a partial emission pump. Proceedings of the ASME 2014 4th Joint US-European Fluids Engineering Division Summer Meeting (FEDSM2014-21418), Chicago, Illinois, USA, August 3-7, 2014: 1-6.

[21] B J Zhao, Y Wang, H L Chen, et al. Impact of wall roughness on the flow law in chamber of a centrifugal pump at off-design operating condition. Journal of Engineering Thermophysics, 2015, 36(9): 1927-1932. (in Chinese)

[22] W Li, W D Shi, X P Jiang, et al. Numerical calculation and experimental study of axial force on multistage centrifugal pump. Transactions of the Chinese Society of Agricultural Engineering, 2012, 28(23): 52-59. (in Chinese)

[23] XYYe, J N Hu, J Wang, et al. Numerical and experimental investigation of performance and reliability in new reverse osmosis seawater desalination high pressure pump. Proceedings of the ASME 2013 International Mechanical Engineering Congress and Exposition (IMECE2013-62598), San Diego, California, USA, November 15-21, 2013: 1-9.

[24] Z L Liu, R He, Y Fan. Fluid leakage characteristics test on the balance cavity of floating impeller. Transactions of the Chinese Society for Agricultural, 2011, 42(9): 113-115. (in Chinese)

[25] W Dong, W L Chu. Numerical analysis and validation of fluid pressure in the back chamber of centrifugal pump. Journal of Mechanical Engineering, 2016, 52(4): 165-170. (in Chinese) 